\title{
Análise dos Fundos de Investimento brasileiros através do CAPM Cointegrado com regimes de Markov
}

\section{Analysis of the Brazilian Equity Funds using the co-integrated CAPM with Markov regime switches}

\author{
Bruno Milani \\ Universidade Federal de Santa Maria - RS UFSM \\ Faculdade Palotina de Santa Maria - FAPAS - RS \\ brunoprofess@gmail.com \\ Paulo Sérgio Ceretta \\ Universidade Federal de Santa Maria - RS UFSM \\ ceretta10@gmail.com
}

Submissão: 04/04/2017

Aprovação: 19/08/2019

\section{RESUMO}

O tradicional modelo CAPM já foi largamente utilizado para analisar a relação entre retornos de fundos de investimento e retornos de mercados. Este artigo analisa a relação entre o retorno de fundos de ações brasileiros e o retorno do Ibovespa, a partir de um modelo análogo ao CAPM, estimado por meio de cointegração de séries temporais. A análise é enriquecida com a estimação de regimes de Markov. Os resultados apontam que há regimes muito bem delineados, tanto no curto quanto no longo prazo, sendo que a dependência dos preços dos fundos em relação aos preços do mercado é maior no período de alta e menor no período intermediário. No curto prazo, o retorno dos fundos depende menos do retorno dos mercados quando o mercado apresenta baixa volatilidade e mais quando o mercado apresenta volatilidade média.

Palavras-Chave: Fundos de Investimento, Cointegração, Regimes de Markov

\begin{abstract}
The traditional CAPM has been widely used to analyze the relationship between mutual funds returns and market returns. This article analyzes the relationship between the equity funds returns and the return of Brazilian Bovespa, with a model analogous to CAPM, estimated by time series cointegration. The analysis is enriched by estimating Markov regime changes. The results show that there are very well-designed regimes, both in the short and long term, and the dependence of prices of funds in relation to market prices is higher when the market is in a high level and low in the middle level market. In the short term, the equity funds returns depends less on market returns when the market shows low volatility and more when the market shows average volatility.
\end{abstract}

Key-words: Equity funds, Co-integration, Markov Switching Model 


\section{INTRODUÇÃO E REFERENCIAL TEÓRICO}

Entre os anos de 1950 e 1960, o mundo das finanças vivenciou uma revolução, protagonizada por Markowitz (1952), Lintner (1965), Treynor (1965), Sharpe (1966) e Jensen (1968), os quais individualmente desenvolveram modelos que estabelecem uma relação teórica entre risco e retorno, estabelecendo um novo paradigma. Até Markowitz (1952) não haviam sido publicados estudos com rigor científico sobre a gestão de portfólios e, embora a preocupação com o retorno do portfólio fosse dominante, não havia a preocupação em definir quais variáveis afetavam ou determinavam o seu retorno. Isso relegou a prática de montagem de portfólios a estratégias de tentativa e erro que visavam encontrar ativos que poderiam obter valorização futura. Markowitz (1952) definiu a alocação ótima de recursos considerando o retorno esperado e sua variância, ou seja, a relação entre risco e retorno.

O investidor individual passou a ser o centro das atenções em Sharpe (1966). Nesse ponto, a existência de uma relação entre risco e retorno estava consolidada e a tarefa do gestor estava firmada em selecionar, entre os inúmeros portfólios possíveis, aquele que seria eficiente.

Contudo, Sharpe (1966) admite que existe a possibilidade de encontrar ativos mal precificados, os quais, ao serem incluídos no portfólio, aumentariam o retorno sem aumentar o risco. Para encontrar tais ativos, seria preciso prever o seu comportamento. Com base nisso, levantou-se a teoria de Random Walks, a qual postula que o comportamento passado de um ativo não possui valor algum para prever seu comportamento futuro.

Dessa maneira, se o comportamento dos ativos for semelhante ao prescrito nesta teoria, encontrar ativos mal precificados pode ser uma tarefa difícil e cara, o que poderia implicar em redução do retorno líquido devido as despesas de corretagem. Nas palavras de Sharpe (1966), "se assim for, não é porque os analistas não fazem seu trabalho corretamente, mas porque o fazem muito bem". Logo, o fato de não se encontrarem ativos mal precificados é um indicador de que o mercado é eficiente.

$\mathrm{O}$ autor destacou destacou-se por ser o primeiro a focar o estudo nos fundos de investimento e levantar o questionamento acerca de quais são suas funções. Um gestor pode gerir um fundo que possui um portfólio mais eficiente do que outro fundo. Porém, estas diferenças não se sustentam no longo prazo, ou seja, um fundo que é superior a outro em um determinado momento não necessariamente continuará sendo. Para avaliar a relação entre risco e retorno passado de fundos de investimento Sharpe (1966) propôs, então, um índice que estabelece a razão entre o retorno médio de uma série e seu desvio-padrão.

Jensen (1968) retomou a discussão sobre a dicotomia de comprar ativos mal precificados na tentativa de prever seu comportamento ou montar um portfólio bem diversificado, direcionando seu estudo à mensuração da capacidade de previsão por parte do gestor. $\mathrm{O}$ autor criticou as medidas de performance criadas anteriormente devido ao fato de todas serem relativas, pois seria mais importante desenvolver uma medida absoluta de desempenho. Em outras palavras, medidas criadas até então, como os índices de Sharpe (1966) e Treynor (1965), classificavam os portfólios (ou fundos de investimento) com o objetivo de compará-los entre si, estabelecendo quais foram os melhores e os desempenhos.

A exposição do retorno do fundo ao retorno do mercado foi compreendida por Jensen (1968) como risco sistêmico. Assim, diferentemente dos modelos que o antecederam, o autor admitiu dois tipos de risco: o risco do portfólio, que pode ser minimizado através da diversificação eficiente; e o risco sistêmico ou risco de mercado, ao qual o portfólio está submetido e que é exógeno à gestão do fundo, ou seja, não depende do comportamento do gestor. Nesse sentido, supondo que o risco do portfólio (posteriormente chamado risco diversificável ou risco idiossincrático) efetivamente seja minimizado, o retorno de um fundo de investimento poderá ser explicado pelo risco sistêmico. 
Mas, seria o risco de mercado o único determinante da performance dos fundos de investimento? Ou ainda, como medir a influência/habilidade do gestor em gerar valor para o cotista? Se existe uma parcela do retorno dos fundos independente da variação do excesso de retorno de mercado, é preciso adir um termo independente na equação, ou seja, um coeficiente linear. Esse coeficiente foi chamado por Jensen (1968) de Alfa $\left(\alpha_{i}\right)$, o qual posteriormente ficou conhecido como Alfa de Jensen, que é o intercepto da reta que explica o retorno dos fundos. A equação é representada na Equação [1].

$$
R_{i, t}-R_{f, t}=\alpha_{i}+\beta_{i}\left(R_{M, t}-R_{f, t}\right)+\varepsilon_{i, t} \text {. }
$$

$\mathrm{Na}$ equação [1], $R_{i, t}$ representa o retorno do fundo $i$ no período $t ; R_{f, t}$ representa o retorno do ativo livre de risco $f$ no período $t ; R_{M, t}$ é o retorno do mercado $M$ no período $t$; $\left(R_{i, t}-R_{f, t}\right)$ é o excesso de retorno do fundo de investimento $i$ no período $t ;\left(R_{M, t}-R_{f, t}\right)$ é o excesso de retorno do mercado $M$ no período $t ; \beta_{i}$ é a medida do risco sistêmico; $\varepsilon_{i, t}$ representa o erro aleatório, $\alpha_{i}$ é o coeficiente linear (Alfa de Jensen), o qual representa "a taxa incremental de retorno do portfólio por unidade de tempo que é devida unicamente a habilidade do gestor em prever o preço futuro de ativos" (JENSEN, 1968).

Esta contribuição foi expressiva, pois finalmente havia uma maneira de avaliar a gestão dos fundos de investimento ou portfólios de maneira absoluta, não dependendo apenas da comparação da performance de seus pares, como em Treynor (1965) e Sharpe (1966). Jensen (1968) também se destacou pela inclusão do risco sistêmico, uma vez que Markowitz (1952) e Lintner (1965) consideravam apenas a existência do risco diversificável. O modelo proposto na Equação [1] consolidou-se como meio para a avaliação de ativos, sob a denominação de Capital Asset Pricing Model (CAPM).

Muito além do tradicional CAPM, a análise do comportamento de ativos financeiros tem avançado com a utilização de novas técnicas, como por exemplo a cointegração, cujo estudo seminal foi o de Engle e Granger (1987), a partir de contribuições de Granger (1966). A utilização da cointegração representa uma considerável mudança, pois ao invés de analisar retornos, ela baseia-se nas séries de preços “em nível”. O seu emprego para a avaliação de portfólios foi preconizado por Alexander (1999), o qual chamou a atenção para o fato de que a diferenciação necessária para a utilização de outros modelos (devido ao problema da nãoestacionaridade) implica em perda de informações referentes as tendências de longo prazo.

Até o surgimento deste método, a análise financeira estava condicionada ao emprego da correlação entre séries temporais, que embora tenha proximidade com o conceito de cointegração, pode levar a conclusões diferentes. Segundo Alexander (1999), a correlação reflete comovimentos nos retornos, os quais são suscetíveis a instabilidades ao longo do tempo. Isso faz com que estratégias de gestão de portfólios baseadas na correlação requeiram frequentes rebalanceamentos. No entanto, a cointegração mensura comovimentos de longo prazo nos preços, que podem ocorrer mesmo com a presença de períodos em que a correlação estática apresenta-se muito baixa.

Alexander e Dimitriu (2004) argumentaram que modelos de otimização para a replicação de benchmarks focam na minimização da variação do tracking error, o qual, por sua vez, representa quanto a variação do retorno do portfólio se afasta da variação do retorno do benchmark. Além disso, há uma preocupação muito forte com os custos de transação envolvendo o rebalanceamento do portfólio. Contudo, modelos de otimização baseados no tracking error ou na correlação sofrem uma série de inconvenientes, especialmente quando aplicados a um cenário de investimentos passivos. Em primeiro lugar, minimizar o tracking error em relação a um índice, sendo uma combinação linear de preços de ações, inclui uma quantidade significativa de ruídos que podem resultar num portfólio com características muito específicas da amostra e instabilidade em situações de grande volatilidade de mercado. 
Ainda de acordo com Alexander e Dimitriu (2004), limitações adicionais são geradas pela natureza da correlação como uma medida de variação: só é aplicável a variáveis estacionárias, tais como retornos de ações, que requerem transformações em relação a variável em nível e levam à desvantagem da perda de informações valiosas como, por exemplo, as tendências comuns nos preços. Por essas razões, a correlação seria uma estatística instável e de curto prazo.

Enfim, Alexander e Dimitriu (2004) criticam o fato de a correlação, dependendo do modelo utilizado para a estimação, ser muito sensível a presença de outliers, nãoestacionaridade e clusters de volatilidade, os quais limitam o uso de séries grandes e podem levar a conclusões errôneas sobre a natureza das dependências de curto prazo. Em suma, estratégias de investimentos baseadas apenas na correlação e na volatilidade podem não garantir a mensuração adequada da performance no longo prazo.

A principal justificativa para a aplicação da cointegração a um conjunto de preços de ações é o fato de eles poderem compartilhar uma tendência estocástica comum (Stock e Watson, 1991). Adicionalmente, Alexander e Dimitriu (2004) destacaram que a cointegração existe quando há pelo menos uma combinação linear estacionária dos seus preços, ou simplesmente, quando há uma reversão à média nos seus spreads. Esta última conclusão não apresenta nenhuma informação sobre a previsão de preços individuais no sistema, ou sobre a deste último em algum momento futuro, mas fornece a informação valiosa de que, independentemente da posição do sistema, os preços manter-se-ão unidos no longo prazo.

A regressão de uma série temporal com raiz unitária contra outra série temporal com raiz unitária pode produzir uma regressão espúria, a menos que o erro gerado por esta regressão seja estacionário (TSAY, 2010, p. 430). Em síntese, é possível que a combinação linear de duas séries não-estacionárias faça com que suas tendências estocásticas se anulem, caracterizando uma relação estável de longo prazo. A situação pode ser exemplificada com o auxílio da Equação [2].

$Y_{t}=\beta_{1}+\beta_{2} X_{t}+\mu_{t}$.

Nessa equação $Y_{t}$ e $X_{t}$ são variáveis em nível (preços); $\beta_{1}$ e $\beta_{2}$ são parâmetros; $\mu_{t}$ é o erro da regressão, que é estacionário.

Ainda de acordo com Tsay (2010, p. 432), no curto prazo pode haver desequilíbrio entre duas séries cointegradas (erro de equilíbrio), mas no longo prazo o desequilíbrio é corrigido pelo mecanismo de correção de erro. Desse modo, o erro da Equação [2], $\mu_{t}$, liga o comportamento de curto prazo de $Y$ com seu valor de longo prazo, realizando uma correção dos desequilíbrios de curto prazo. O mecanismo de correção de erro mostra que $Y$ depende de $X$ e também do erro do período anterior, conforme Equação [3].

$\left\{\Delta Y_{t}=\alpha_{0}+\alpha_{1} \Delta X_{t}+\alpha_{2} \mu_{t-1}+\varepsilon_{t}\right.$

$\left\{\Delta X_{t}=\alpha_{0}+\alpha_{1} \Delta Y_{t}+\alpha_{2} \mu_{t-1}+\varepsilon_{t}\right.$.

Em que $\Delta Y_{t}$ é a variação do preço de $Y_{t} ; \Delta \mathrm{X}_{\mathrm{t}}$ é a variação do preço de $\mathrm{X}_{\mathrm{t}} ; \alpha_{0}, \alpha_{1}$ e $\alpha_{2}$ são parâmetros; $\mu_{\mathrm{t}-1}$ é o erro da Equação [2] defasado em um período; $\varepsilon_{\mathrm{t}}$ é o erro.

Segundo Tsay (2010, p. 432), a equação [3] demonstra que $\Delta \mathrm{Y}_{\mathrm{t}}$ depende de $\Delta \mathrm{X}_{\mathrm{t}} \mathrm{e}$ também de um termo de erro de equilíbrio. Se $\Delta \mathrm{X}_{\mathrm{t}}$ é zero e $\mu_{\mathrm{t}-1}$ é positivo, então $\mathrm{Y}_{\mathrm{t}}$ está elevado demais para estar em equilíbrio. Espera-se que $\alpha_{2}$ seja negativo e, portanto, $\alpha_{2} \mu_{\mathrm{t}-1}$ deve ser negativo, de forma que $\Delta \mathrm{Y}_{\mathrm{t}}$ será negativo para restaurar o equilíbrio. Em resumo, se $\mathrm{Y}_{\mathrm{t}}$ estiver acima de seu valor de equilíbrio, ele começará a cair no período seguinte para corrigir o erro de equilíbrio, daí o nome de Mecanismo de Correção de Erro. Assim, o mecanismo de correção de erro faz a correção dos desequilíbrios de curto prazo no sistema de equações representado por [3], seja quando $\Delta X_{t}$ ou quando $\Delta Y_{t}$ é a variável dependente.

Levando em consideração que preços são processos com memória de longo prazo (Granger e Terasvirta, 1993), a cointegração é capaz de explicar o seu comportamento de 
longo prazo. De acordo com Alexander e Dimitriu (2004), a lógica da construção de portfólios baseada no relacionamento cointegrado com um benchmark encontra suporte em dois aspectos principais: em primeiro lugar, a diferença de preços entre um benchmark e o portfólio é, por definição, estacionária e implica que o tracking portfólio vai estar "amarrado" ao benchmark no longo prazo; em segundo lugar, os pesos das ações, baseados num longo histórico de preços, terão estabilidade apurada. Estas propriedades são o resultado do uso completo da informação contida nos preços das ações antes de remover sua tendência.

No entanto, há a possibilidade de que o vetor de cointegração nem sempre se mova para o equilíbrio de longo prazo, apenas quando o desvio do equilíbrio exceda um threshold crítico. Esta possibilidade foi levantada por Balke e Fomby (1997), que argumentam que a cointegração pode ser "ativada" ou "desativada" conforme a variação dos preços.

Balke e Fomby (1997) examinaram as séries de retorno dos títulos públicos dos EUA e suas taxas de desconto, verificando a existência de cointegrações com thresholds, que são limites dentro dos quais diferentes regimes podem acontecer, como se fosse necessário um modelo diferente para explicar as variações dos preços dentro cada regime. Os resultados obtidos foram positivos, comprovando que não existe cointegração dentro de uma determinada amplitude de preços, mas somente fora dela. Assim, eles atestaram a importância da análise dos thresholds, pois ela permite compreender melhor o comportamento dos preços, sabendo que a relação entre duas variáveis altera-se em diferentes condições de mercado.

Os thresholds não são, contudo, a única maneira de averiguar mudanças de regime. Os regimes de Markov enfocam, ao invés do uso de limites, o uso da probabilidade para localizar trocas de regime em séries temporais não lineares. Esta ideia é discutida por Tong (1983) e operacionalizada por Hamilton (1989) através de um modelo autoregressivo com mudanças de regime aperiódicas. Segundo Tsay (2010, p. 660), uma série temporal apresenta duas mudanças de regimes de Markov caso satisfaça a Equação [4].

$x_{t}=\left\{\begin{array}{lll}c_{1}+\sum_{i=1} p \emptyset_{1, i} x_{t-1}+a_{1, t} & \text { se } & S_{t}=1 \\ c_{2}+\sum_{i=1} p \emptyset_{2, i} x_{t-1}+a_{2 t} & \text { se } & S_{t}=2 .\end{array}\right.$

Em que $S_{\mathrm{t}}$ assume valores 1 ou 2 em uma cadeia de Markov de primeira ordem, com probabilidades de transição dadas pelas Equações [5], [6], [7] e [8]:

$P\left(S_{t}=1 \mid S_{t-1}=1\right)=1-w_{1}$,

$P\left(S_{t}=2 \mid S_{t-1}=2\right)=1-w_{2}$.

$P\left(S_{t}=2 \mid S_{t-1}=2\right)=1-w_{2}$

$P\left(S_{t}=2 \mid S_{t-1}=2\right)=1-w_{2}$

As séries de inovação $a_{1, t}$ e $a_{2 t}$ são sequências de ruído branco e independentes entre si. Um coeficiente $\mathrm{w}_{1}$ pequeno significa que o modelo tende a permanecer no estado $i$. Na verdade, $1 / w_{i}$ é a expectativa de duração do processo que permanece no estado $i$. Por definição, o modelo Markov Switching autoregressive (MAS) usa uma cadeia de Markov oculta para governar a transição de uma função de média condicional para outra. Dessa forma, neste modelo nunca há certeza sobre qual estado $x_{t}$ pertence. Tsay (2010, p. 661) ainda argumenta que o modelo MSA pode facilmente ser generalizado para casos com mais de dois estados.

Considerando as importantes vantagens comparativas de usar a cointegração ao invés da correlação para a montagem de portfólios de ativos, seria possível, caso encontrado, explorar um relacionamento de longo prazo entre preços de ativos e índices de mercados, com o objetivo de construir estratégias de compra e venda de ações. Isso é evidenciado por Alexander et al. (2002) que investigam a performance de diferentes estratégias long-short desenvolvidas no universo das ações do SP100. Sua aplicação implicou uma extensa pesquisa 
acerca de um grande número de portfólios baseados nas relações de cointegração e otimizações com diferentes parâmetros do modelo - como período de treinamento, meta de tracking error e números de ativos no portfólio - para a melhor combinação long-short. Seus resultados, mesmo baseados num algoritmo de caixa-preta, indicam que a otimização da cointegração pode garantir um coeficiente linear estável, com baixa volatilidade e nãocorrelacionado com os retornos do mercado.

Dessa forma, os autores utilizaram uma equação análoga ao CAPM, mas com a inclusão de um vetor de correção de erros, de forma que o coeficiente linear gerado será significativo se houver uma relação de longo prazo entre as variáveis. O Alfa oriundo de um CAPM cointegrado foi considerado mais apropriado para analisar a performance em séries muito longas, pois o vetor de correção de erros ajusta as discrepâncias transitórias entre os retornos dos fundos de investimento e o mercado.

Maurer (2008) comparou estratégias de index tracking construídas com base na cointegração de preços de ativos e outra com base na montagem de uma carteira otimizada continuamente, sob a premissa de equilíbrio de mercado. Ambas as estratégias são capazes de seguir um índice, mantendo consistência ao analisar pequenas sub-amostras. A estratégia da cointegração é preferida, especialmente ao se analisar o mercado britânico, pois há evidências fora da amostra de que as diferenças entre os log-retornos dos índices e dos portfólios de ações seguem um processo estacionário.

Chu (2010) analisou a cointegração dos fundos de investimento do sistema previdenciário oficial de Hong Kong no período de 2001 a 2008. Seus resultados apontaram que 56,43\% dos fundos têm seus preços cointegrados com o índice de mercado e que alguns fundos, embora apresentem comovimentos no curto prazo, não os apresentam no longo prazo. Isso indica que os gestores de fundos montam estratégias para tentar vencer o mercado. Ademais, a cointegração de fundos dos Estados Unidos e do Japão com o índice Hang Seng também foi testada, porém rejeitada, evidenciando os benefícios da diversificação internacional.

Kim (2011) examinou a cointegração entre mercados financeiros dos Estados Unidos e do leste da Ásia e nove Exchange Traded Funds (ETFs) de 2004 a 2010, com dados de frequência diária, divididos em sub-amostras referentes ao período anterior e posterior à crise do subprime. As evidências apontam a existência de cinco fundos cointegrados tanto antes quanto depois da crise e a existência de dois fundos que não eram cointegrados antes, mas passaram a ser depois.

Objetivo deste estudo é verificar se há relação de longo prazo entre os fundos de investimento brasileiros e o mercado e se existe um coeficiente linear, ou seja, um Alfa de Jensen significativo nesta relação, valendo-se da técnica de cointegração com mudanças de regime de Markov. A Seção 2 apresentará o método e os dados utilizados.

\section{MÉTODO E DADOS}

O presente estudo conta com dados fornecidos pela Associação Nacional das Entidades dos Mercados Financeiro e de Capitais (ANBIMA), em frequência diária, através do software SI-ANBID 4.2, do qual foram extraídas as séries temporais de patrimônio líquido e retorno ponderado pelo patrimônio líquido dos fundos de investimento de gestão passiva e ativa cujos benchmarks são o Ibovespa e o IBrX, além dos fundos "livres", ou seja, aqueles que não são atrelados a um benchmark específico nem definem explicitamente um tipo de gestão.

Estas séries foram importadas para o software Microsoft Excel, no qual foi calculado o seu retorno médio, ponderado pelos seus patrimônios líquidos. Assim, trabalhou-se com uma 
única série temporal para representar o retorno dos fundos de investimento em ações brasileiros.

A série do índice Ibovespa foi obtida no site da Bolsa de Mercadorias e Futuros BOVESPA (BM\&FBOVESPA), sendo seu retorno posteriormente calculado. O período compreendido pela amostra vai de 02/05/2001 a 17/02/2012, com observações de frequência diária. O período foi escolhido por ser o mais abrangente possível, totalizando 2678 observações.

Devido à natureza da técnica de cointegração, a qual só permite a utilização de variáveis em nível, as séries de retornos dos fundos e do Ibovespa passaram foram transformadas para um sistema de pontos que serviu como um artifício para simular uma série de preços. Foi arbitrado que no dia 01/05/2001, o dia antes do início da série, o "preço" do retorno dos fundos e do Ibovespa era 100. A Equação [9] demonstra como o cálculo do preço artificial foi realizado.

$P_{t}=P_{t-1} *\left(1+\frac{R_{t}}{100}\right)$.

Em que $P_{t}$ é o preço artificial no período $t ; \mathrm{R}_{\mathrm{t}}$ é o retorno percentual no período $t$.

Para analisar a relação de longo prazo entre o preço de fundos de investimento e do índice Ibovespa, utilizou-se a técnica de cointegração, de acordo com Tsay (2010, p. 432) e Equações [2] e [3]. Assim, construiu-se um modelo análogo ao CAPM, de acordo com Jensen (1968) e com a Equação [1], com a inclusão de um vetor de correção de erros, conforme proposto por Alexander et al. (2002). As Equações [10] e [11] representam o modelo utilizado.

$F_{t}=\beta_{0}+\beta_{1} M_{t}+\mu_{t}$

$\left\{\Delta F_{t}=\alpha_{0}+\alpha_{1} \Delta M_{t}+\alpha_{2} \mu_{t-1}+\varepsilon_{1, t}\right.$

$\left\{\Delta M_{t}=\alpha_{0}+\alpha_{1} \Delta F_{t}+\alpha_{2} \mu_{t-1}+\varepsilon_{2, t}\right.$

Em que $\mathrm{F}_{\mathrm{t}}$ é o preço dos fundos no período $t ; \mathrm{M}_{\mathrm{t}}$ é o preço do índice Ibovespa no período $t ; \beta_{0}$ e $\beta_{1}$ são parâmetros; $\mu_{\mathrm{t}}$ é o erro estacionário; $\alpha_{0}$ é o coeficiente linear análogo ao Alfa de Jensen (1968); $\alpha_{1}$ é a sensibilidade do retorno dos fundos ao retorno do mercado; $\mu_{\mathrm{t}-1}$ é o vetor de correção de erros; $\varepsilon_{\mathrm{t}}$ é o erro da Equação [11].

$\mathrm{O}$ relacionamento entre preços dos fundos e preços do mercado representado pela Equação [10] pode ser entendido como dinâmico no longo prazo, pela sua natureza, pois a série de preços é um acumulado de choques de curto prazo no decorrer de um período maior. O sistema de Equações [11], por tratar-se do cálculo da elasticidade, representa a dinâmica de curto prazo entre o retorno dos fundos de investimento e o retorno do mercado. O sistema de Equações [11] e a Equação [10] serão estimados em regimes de Markov, conforme probabilidades de transição definidas pelas Equações [5], [6], [7] e [8]. As estimações e probabilidades de transição de regime serão calculadas pelo software R, utilizado para a estimação dos parâmetros. A Seção 3 trará a análise dos resultados obtidos.

\section{RESULTADOS}

Dando início à análise dos resultados, a Tabela 1 apresenta a estimativa de relacionamento de longo prazo, a partir da Equação [10], a qual resultou na identificação de três diferentes regimes $(0,1$ e 2$)$.

Tabela 1. Estimativa do relacionamento de longo prazo com três regimes de Markov

\begin{tabular}{lrrrr}
\hline Parâmetro Coeficiente & Erro padrão & Teste t & P-valor \\
\hline$\beta_{0}(0)$ & 0,653 & 0,017 & 38,100 & 0,000 \\
$\beta_{0}(1)$ & 1,460 & 0,012 & 119,000 & 0,000 \\
$\beta_{0}(2)$ & 0,339 & 0,008 & 43,700 & 0,000
\end{tabular}




\begin{tabular}{lllrl}
$\beta_{1}(0)$ & 0,784 & 0,003 & 245,000 & 0,000 \\
$\beta_{1}(1)$ & 0,671 & 0,002 & 313,000 & 0,000 \\
$\beta_{1}(2)$ & 0,887 & 0,001 & 644,000 & 0,000 \\
$\mu_{t}(0)$ & 0,060 & 0,002 & 35,700 & 0,000 \\
$\mu_{t}(1)$ & 0,039 & 0,001 & 37,700 & 0,000 \\
$\mu_{t}(2)$ & 0,020 & 0,000 & 43,300 & 0,000 \\
\hline
\end{tabular}

log-likelihood 5310,858 AIC -3,955

Teste de lineariedade LR Chi^2(12) $=6958,1$ p-valor $(0,000)$

A Tabela 1 demonstra as três equações estimadas, uma para cada um dos três regimes existentes (regimes 0,1 e 2). O Regime 1 apresenta constante $\beta_{0}$ alta e coeficiente de covariância $\beta_{1}$ mais baixo, indicando que, quando a série entra neste regime, a suscetibilidade à variação do preço do mercado diminui. O Teste de linearidade LR rejeita a hipótese nula de que há somente um regime. Os valores de $\mu_{t}$ referem-se à variância do erro em cada regime.

O Regime 2, por sua vez, apresenta a situação contrária: o coeficiente linear é muito pequeno, embora significativo, enquanto a suscetibilidade à variação do mercado é muito alta, evidenciando que neste regime o preço dos fundos está muito sujeito ao preço do mercado. Dentro da interpretação tradicional proposta por Jensen (1968), em todos regimes o gestor consegue auferir ganhos superiores ao mercado, mas no Regime 1 consegue muito mais do que no Regime 2. Neste contexto, o Regime 0 é um intermediário, com $\beta_{0}$ menor que o Regime 1 e maior que o Regime 2, bem como $\beta_{1}$ maior que o Regime 1 e menor que o Regime 2. Embora existam diferenças nos valores dos coeficientes, todos apresentaram pvalor significativo ao grau de significância de $1 \%$.

A Tabela 2 apresenta a matriz de probabilidades de transição de regimes.

Tabela 2. Matriz das probabilidades de transição

\begin{tabular}{llll}
\hline & \multicolumn{1}{l}{ Regime (0,t) } & Regime (1,t) & Regime (2,t) \\
\hline Regime (0,t+1) & 0,995 & 0,003 & 0,002 \\
Regime (1,t+1) & 0,002 & 0,991 & 0,004 \\
Regime (2,t+1) & 0,003 & 0,006 & 0,994 \\
\hline
\end{tabular}

Análise do resíduo do relacionamento de longo prazo

Teste Dickey-Fuller Aumentado (GLS) -3.023 (p-valor =0.002) [Rejeita raiz unitária]

Teste KPSS 0.337 (valor crítico $5 \%=0.347$ ) [Não rejeita estacionariedade]

A Tabela 2 apresenta as probabilidades de ocorrerem trocas de regime. Uma vez no Regime 0, a probabilidade de manter-se neste regime é de 99,5\%. Quando no Regime 1, a probabilidade de manter-se no mesmo regime é de 99,1\%. De forma semelhante, se a série estiver no Regime 2, há 99,4\% de chances de permanecer nele. Assim, verifica-se que existe a tendência dos regimes se manterem, de forma que não se espera alterações com muita frequência. Os testes de Dickey-Fuller e KPSS evidenciam que a série é estacionária, ao grau de significância de 5\%. A Figura 1 demonstra graficamente as transições de regimes.

Figura 1. Índice ponderado dos fundos de ações e os regimes de Markov 


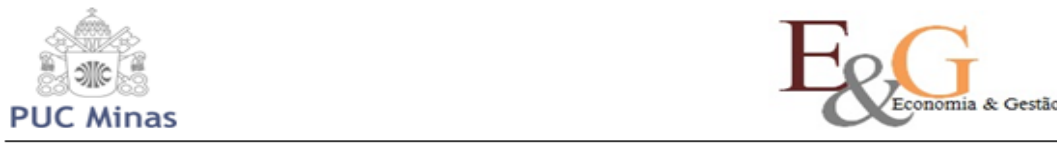

\section{ISSN 1984-6606}

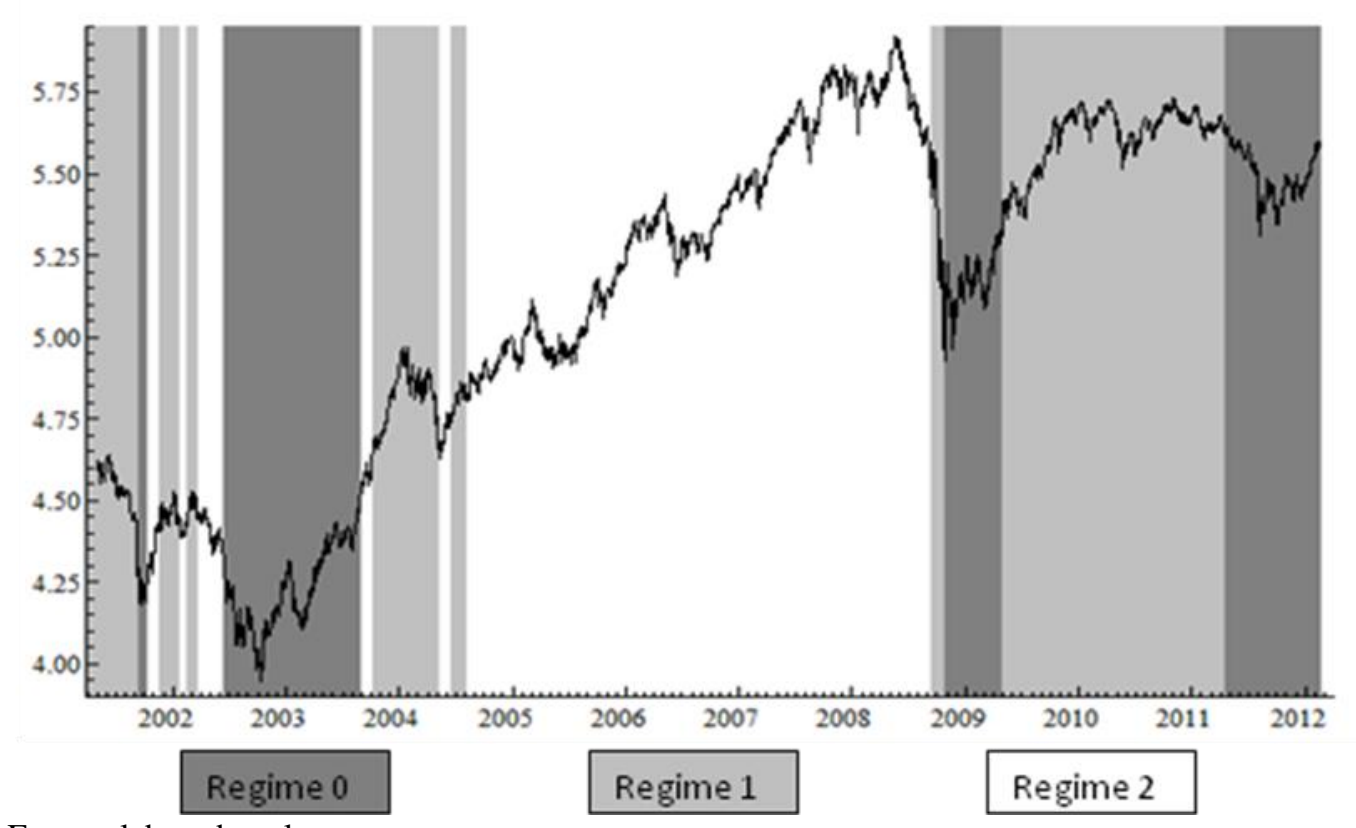

Fonte: elaborado pelos autores

A Figura 1 demonstra claramente que o Regime 2 prevalece em momentos em que o mercado está subindo, enquanto o Regime 0 é o regime crítico, ou seja, é o regime que acontece quando o mercado está com os preços subvalorizados em relação aos períodos próximos. O Regime 1 é o intermediário entre os Regimes 0 e 2. Percebe-se que o regime crítico aparece entre o final de 2002 e o início de 2003 com o efeito da incerteza gerada pela mudança de governo; em 2009, com a crise do subprime; em 2011, sinalizando econômica nos países europeus.

Tomando por base os coeficientes apresentados na Tabela 1 e os regimes identificados na Figura 1, verifica-se que no Regime 2, que é o regime de crescimento do mercado, os preços dos fundos estão mais suscetíveis ao preço do mercado do que nos demais regimes, tendo em vista que seu $\beta_{1}$ é mais alto e que seu $\beta_{0}$ é menor. No momento em que o mercado vivencia uma situação de expansão, o preço dos fundos depende mais do preço do mercadok.

No Regime 1, que é o regime intermediário, os coeficientes $\beta_{0}$ e $\beta_{1}$ apontam que o preço dos fundos depende menos do preço do mercado, pois a constante $\beta_{0}$ é muito alta e a suscetibilidade do fundo ao mercado, $\beta_{1}$, é muito baixa. Os resultados, portanto, indicam que em momentos de crescimento, representados pelo Regime 2, os fundos acompanham o mercado, o que pode ser considerado um bom indicador. Já nos momentos em que o mercado encontra-se em situação intermediária (Regime 1), caracterizando uma situação de incerteza, os fundos protegem o investidor, fazendo com que o preço dos fundos dependa menos do preço do mercado. Isso ficou evidente devido ao baixo coeficiente de $\beta_{1}$ e ao alto coeficiente de $\beta_{0}$.

Contudo, nos momentos em que o mercado está em decadência (Regime 0) o fundo não consegue se desvencilhar tanto do mercado como no Regime 1 , tendo em vista os valores medianos de $\beta_{0}$ e $\beta_{1}$. No Regime 0 , que é o regime crítico, o coeficiente $\beta_{1}$ é menor do que no Regime 2 e maior do que no Regime 1 . O coeficiente $\beta_{0}$ é maior do que no Regime 2 e menor do que no Regime 1. Justamente quando o fundo mais necessita "fugir" da oscilação do mercado, o gestor não consegue resultado satisfatório, apesar de ser natural o aumento da dependência com o mercado em momentos turbulentos.

A Tabela 3 traz as estimativas para os coeficientes da Equação [11], que representa um modelo CAPM cointegrado análogo ao CAPM, conforme propõe Alexander et al. (2002), sujeito às mudanças de regime de Markov. 
Tabela 3. Estimativa da dinâmica de curto prazo com três regimes de Markov

\begin{tabular}{|c|c|c|c|c|}
\hline & Coeficiente & $\begin{array}{r}\text { Erro padrão } \\
\text { robusto }\end{array}$ & Teste $\mathbf{t}$ & P-valor \\
\hline$\alpha_{0}(0)$ & $-0,067$ & 0,05 & $-0,94$ & 0,826 \\
\hline$\alpha_{0}(1)$ & 0,031 & 0,006 & 4,37 & 0,000 \\
\hline$\alpha_{0}(2)$ & 0,093 & 0,016 & 5,52 & 0,000 \\
\hline$\alpha_{1}(0)$ & 0,662 & 0,019 & 25,10 & 0,000 \\
\hline$\alpha_{1}(1)$ & 0,726 & 0,004 & 109,00 & 0,000 \\
\hline$\alpha_{1}(2)$ & 0,373 & 0,012 & 14,40 & 0,000 \\
\hline$\mu_{\mathrm{t}-1}(0)$ & 6,869 & 1,45 & 3,96 & 0,000 \\
\hline$\mu_{\mathrm{t}-1}(1)$ & 0,296 & 0,179 & 1,48 & 0,069 \\
\hline$\mu_{\mathrm{t}-1}(2)$ & 1,722 & 0,387 & 3,33 & 0,000 \\
\hline$\varepsilon(0)$ & 0,972 & 0,05 & 6,89 & 0,000 \\
\hline$\varepsilon(1)$ & 0,182 & 0,006 & 18,20 & 0,000 \\
\hline$\varepsilon(2)$ & 0,555 & 0,016 & 18,20 & 0,000 \\
\hline
\end{tabular}

Coeficientes obtidos pela estimação conforme Equação [9], log-likelihood-1622,54 AIC 1,247

Linearity LR-test $\mathrm{Chi}^{\wedge} 2(12)$ 2108,900 0,000

A Tabela 3 evidencia que a constante $\alpha_{0}$ não foi significativa para o Regime 0 e que o termo de correção de erros $\left(\mu_{t-1}\right)$ não foi significativo no Regime 1 , tendo em vista seus pvalores superiores a 0,05 . Logo, no Regime 0 o coeficiente $\alpha_{1}$, que representa a sensibilidade do retorno do fundo ao retorno do mercado é alto, mas não tanto quanto o $\alpha_{1}$ do Regime 1 . No Regime 1 há um $\alpha_{0}$ muito pequeno e o seu $\alpha_{1}$ é o mais alto entre os três regimes. No Regime 2 , o $\alpha_{0}$ é um pouco mais alto que o do Regime 1 , mas seu $\alpha_{1}$ é muito menor. O teste de linearidade rejeita a hipótese nula de que há somente um regime. Os coeficientes $\varepsilon$ representam a variância do erro em cada regime.

Neste contexto, o coeficiente linear $\alpha_{0}$ é análogo ao Alfa de Jensen (1968), uma medida de performance amplamente conhecida. Mesmo que o vetor de correção de erros não tenha sido significativo no Regime 1, o modelo gerado difere do tradicional CAPM, pois a inserção do vetor de correção de erros no modelo impactou a estimação dos demais coeficientes. A Tabela 4 apresenta as probabilidades de transição de regime do modelo estimado pela Equação [3].

Tabela 4. Matriz das probabilidades de transição

\begin{tabular}{llll}
\hline & Regime (0,t) & Regime $(\mathbf{1 , t})$ & Regime (2,t) \\
\hline Regime $(0, t+1)$ & 0,963 & 0,004 & 0,012 \\
Regime $(1, t+1)$ & 0,009 & 0,996 & 0,000 \\
Regime $(2, t+1)$ & 0,027 & 0,000 & 0,988 \\
\hline
\end{tabular}

A Tabela 4 deixa claro que, de maneira similar a matriz de probabilidades de transição de regime evidenciada pela Tabela 1 , as probabilidades de que os regimes permaneçam os mesmos são muito altas. Em resumo, não se espera que os regimes variem constantemente. A Figura 2 apresenta o gráfico dos log-retornos do índice ponderado dos fundos de ações, sob o prisma dos regimes de Markov. 
Figura 2. Log-variações do índice ponderado dos fundos de ações e os regimes de Markov

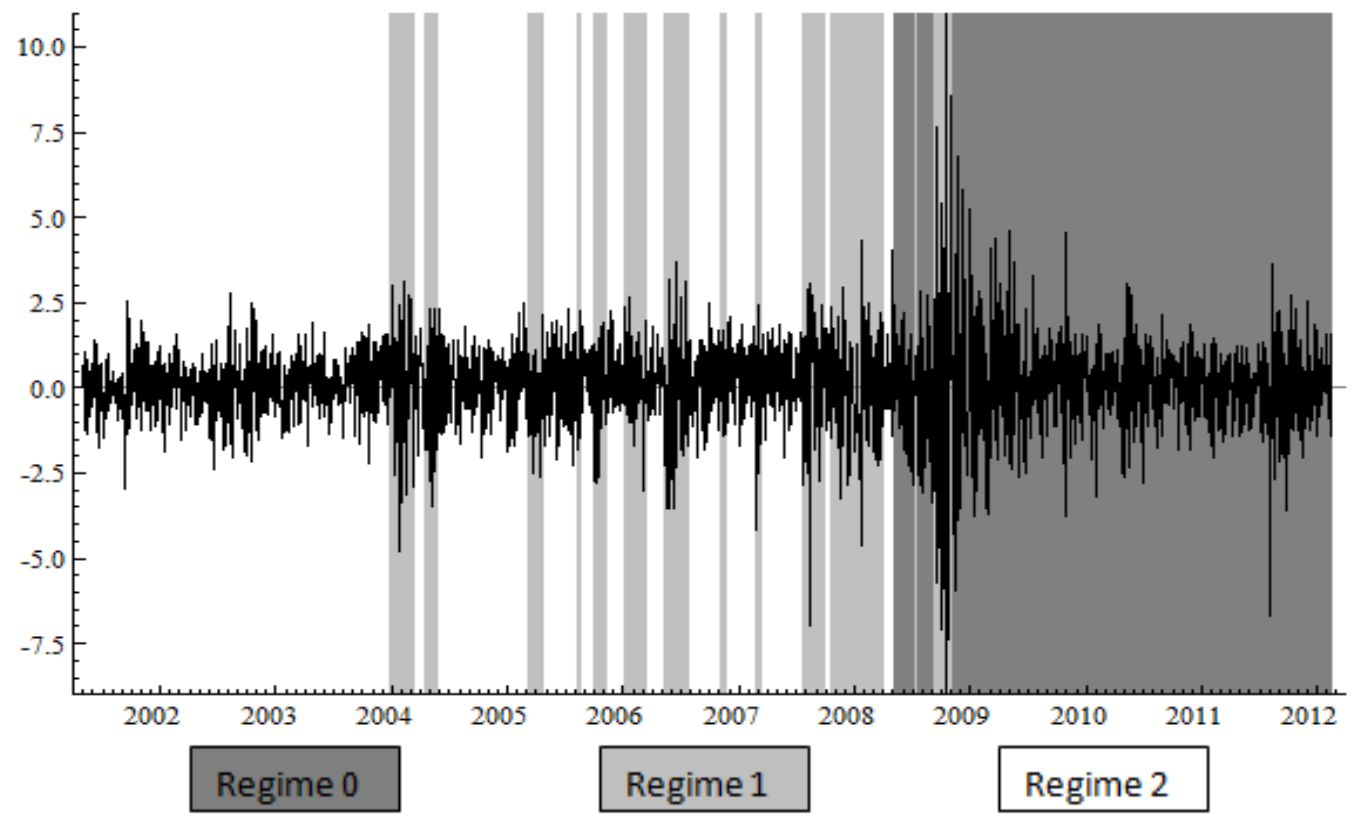

Fonte: dados elaborados pelos autores

A Figura 2 demonstra claras diferenças entre os regimes: o Regime 2 ocorre em momentos de baixa volatilidade; o Regime $1 \mathrm{em}$ momentos de volatilidade intermediária; e o Regime 0 em momentos recentes de muita volatilidade, que podem ser interpretados como momentos críticos. Retomando a discussão acerca dos coeficientes apresentados na Tabela 2, percebe-se que durante o Regime 0 , de volatilidade elevada, o fundo apresenta performance inferior (segundo modelo de Jensen). No Regime 2, de volatilidade mais baixa, os fundos apresentaram o maior coeficiente linear, indicando que o gestor do fundo consegue gerar mais retorno para o cotista em momentos mais estáveis.

O coeficiente $\alpha_{1}$ foi menor no Regime 2, de volatilidade reduzida, ao passo que foi mais alto nos Regimes 0 e 1 . Isso significa que em períodos de baixa volatilidade o gestor expõe menos o fundo ao mercado e gera retorno de outra forma. Já em períodos de volatilidade elevada, o fundo fica mais exposto ao mercado e o coeficiente linear decai. Contudo, estes resultados não parecem ser satisfatórios para o cotista, uma vez que não seria vantajoso para ele ter seu patrimônio exposto acentuadamente ao mercado em períodos críticos, da mesma forma que poderia ser vantajoso um $\alpha_{1}$ um pouco mais alto em períodos de baixa volatilidade, em que há mais previsibilidade.

$\mathrm{Na}$ verdade, a relação entre $\alpha_{0}$ e $\alpha_{1}$, ou seja, a relação entre o Alfa de Jensen e a exposição ao mercado, não é suficientemente compreendida, porque é possível gerar um Alfa elevado com pouca exposição ao mercado, fazendo com que o retorno seja inferior a um modelo com Alfa nulo e exposição muito alta ao mercado. Com base nos coeficientes estimados apresentados nas Tabelas 1 e 3, foi realizada a simulação exposta na Tabela 5, que calcula o retorno do fundo nos três regimes estudados, cada um em três cenários: quando o retorno do mercado é -1; quando o retorno do mercado é 0 ; e quando o retorno do mercado é 1. A primeira coluna evidencia qual o regime em questão. A segunda coluna representa o retorno do mercado $\left(\Delta M_{t}\right)$. A terceira coluna representa o coeficiente linear $\left(\beta_{1}\right)$, que é nulo no regime 0 por não ter sido significativo. A quarta coluna identifica o coeficiente angular $\left(\beta_{2}\right)$, que no caso é a exposição do fundo ao mercado. A última coluna representa o resultado do retorno do Fundo de Investimento, tendo em vista os coeficientes de cada regime.

Tabela 5. Simulação do resultado para o cotista em três cenários 


\begin{tabular}{lrrrr}
\hline Regime & $\Delta M_{t}$ & $\beta_{1}$ & $\beta_{2}$ & \multicolumn{1}{c}{$\Delta F_{t}$} \\
\hline Regime 0 & -1 & 0 & 0,662 & $-0,662$ \\
& 0 & 0 & 0,662 & 0,000 \\
& 1 & 0 & 0,662 & 0,662 \\
\hline \multirow{2}{*}{ Regime 1 } & -1 & 0,031 & 0,726 & $-0,695$ \\
& 0 & 0,031 & 0,726 & 0,031 \\
& 1 & 0,031 & 0,726 & 0,757 \\
\hline \multirow{2}{*}{ Regime 2 } & -1 & 0,093 & 0,373 & $-0,280$ \\
& 0 & 0,093 & 0,373 & 0,093 \\
& 1 & 0,093 & 0,373 & 0,466 \\
\hline
\end{tabular}

A Tabela 5 apresenta uma simples simulação do resultado obtido pelo cotista em cada um dos três regimes esperados, conforme coeficientes estimados apresentados nas Tabelas $1 \mathrm{e}$ 3. Três situações são apresentadas: retorno do mercado igual a $-1 \%$; retorno do mercado nulo; retorno do mercado igual a $1 \%$. O retorno do fundo é calculado multiplicando o retorno do mercado pelo coeficiente $\beta_{2}$ e somando o coeficiente $\beta_{1}$.

Ao tomar como medida de performance o Alfa de Jensen (1968), seria comum afirmar que o Regime 2 oferece o melhor retorno ao cotista, tendo em vista seu maior coeficiente linear. Contudo, em momentos em que o mercado apresenta retorno positivo, a baixa exposição representa um prejuízo, como pode ser verificado no resultado de $0,466 \%$ obtido no Regime 2, na situação em que o mercado apresenta retorno de 1\%. Essa é a pior performance entre todos os regimes nesta situação de mercado, embora seja imprescindível levar em consideração que o Regime 2 é também o menos volátil e, sob este ponto de vista, é esperado menos retorno. A situação em que o mercado oferece retorno de $-1 \%$ encontra neste regime seu melhor resultado: prejuízo de apenas $-0,280 \%$.

Logo, delineia-se uma contradição: o Regime 2 é o menos volátil, apresentando menores retornos em situação de mercado em alta e maiores retornos em situação de mercado em baixa, devido a sua baixa exposição ao mercado. Contudo, seu Alfa de Jensen (1968) é o mais alto entre os três regimes, o que indicaria melhor performance.

O Regime 1, de volatilidade intermediária, apresenta maior retorno simulado quando o mercado apresenta retorno de $1 \%$ e menor retorno simulado quando o mercado apresenta retorno de $-1 \%$. Neste regime, considerado de volatilidade intermediária, o Alfa de Jensen é positivo, mas menor do que o do Regime 2. Por fim, o Regime 0, de alta volatilidade, apresenta Alfa nulo e os resultados para os fundos são intermediários em relação aos obtidos no Regimes 1 e 2.

Resumidamente, no regime 0, que é o mais volátil, o Alfa foi menor. Nos regimes menos voláteis, o Alfa foi o mais alto. A existência de um coeficiente linear significativo está de acordo com o averiguado por Alexander et. al. (2002). A exposição ao mercado, pelo contrário, foi menor em períodos de baixa volatilidade e maior em períodos de alta volatilidade, o que não é um bom indicador, pois faz com que o investidor esteja exposto ao risco justamente quando isto é menos desejável. O fato de existir cointegração entre as séries vai ao encontro de Chu (2010) e Kim (2011), os quais encontraram cointegração entre a maior parte dos fundos de investimento e o mercado. Ao contrário de Balke e Fomby (1997), o presente estudo encontrou cointegração em todos os regimes.

\section{CONSIDERAÇÕES FINAIS}

Partindo dos resultados obtidos por Alexander et al. (2002) e Alexander e Dimitriu (2004), este estudo teve como objetivo verificar a ocorrência de relação estável de longo 
prazo entre o retorno dos fundos de investimento e o retorno do mercado e a existência de um coeficiente linear, ou seja, um Alfa significativo nesta relação. As análises foram incrementadas a partir do uso da técnica de cointegração com mudanças de regime de Markov. A importância da análise da cointegração entre fundos de investimento e seus índices de mercado foi anteriormente verificada por Chu (2010) e Kim (2011).

Junto à ANBIMA foram obtidas as séries de retorno consolidado dos fundos de investimento de gestão passiva e ativa cujos benchmarks são o Ibovespa e o IBrX, além dos fundos livres. A partir delas, foi criada uma série de retornos médios ponderados pelo patrimônio líquido de cada categoria prévia, representando o retorno consolidado dos fundos de investimentos brasileiros no período. O retorno do índice Ibovespa foi utilizado como proxy de mercado. Para a análise de cointegração, as séries de retorno foram transformadas em séries em nível.

Com base nas Equações [10] e [11], um CAPM cointegrado foi estimado, sujeito a mudanças de regimes de Markov, conforme Equações [4], [5], [6], [7] e [8]. Três regimes foram identificados: o Regime 0, que ocorre em momentos de queda; o Regime 1, intermediário; e o Regime 2, que prevalece quando o mercado está se valorizando. As crises da Europa, do Subprime e do início do governo Lula, por exemplo, estão bem definidas dentro do Regime 0.

A regressão das séries em nível (análise de longo prazo), que é interpretável por apresentar resíduo estacionário (GUJARATI, 2006, p. 659), demonstrou que em períodos de queda (Regime 0) a exposição ao mercado é menor do que quando o mercado está se valorizando (Regime 2), além de que o coeficiente linear é maior. Isso significa que o gestor do fundo conseguiu proteger o cotista nos momentos em que o mercado esteve em crise, resultado que pode ser considerado favorável para o investidor. Este resultado é particularmente importante devido à extensa literatura acerca de fundos de investimentos que discute se o gestor do fundo agrega valor ou não, iniciada em Jensen (1968).

Para a análise da dinâmica de curto prazo foi estimada a Equação [11], que insere o termo de correção de erros numa regressão com a primeira diferença das séries. Ele não gerou coeficiente significativo para o Regime 1 . Também ficou evidente que nos regimes de menor volatilidade o coeficiente linear é mais alto e a exposição ao mercado é menor. No Regime 0 sequer há coeficiente linear significativo, enquanto o coeficiente $\alpha_{1}$ é de 0,662 ; No Regime 1 , o coeficiente linear passa para 0,031 e $\alpha_{1}$ aumenta para 0,726 . No Regime 2, o coeficiente linear aumenta para 0,093, enquanto $\alpha_{1}$ diminui para 0,373. Nos períodos de maior volatilidade ( 0 e 1$)$, o retorno dos fundos apresenta a maior dependência em relação ao retorno do mercado e os menores coeficientes lineares. Ou seja, nos momentos críticos os fundos sofrem com maior vinculação ao retorno do mercado, enquanto nos momentos de valorização do mercado perdem a chance de usufruir deste crescimento. Embora estes resultados apresentem uma perspectiva negativa, a análise das séries em nível (análise de longo prazo) não os corrobora, indicando que a fraca performance de curto prazo é compensada pelos resultados de longo prazo. Esta pode ser considerada a principal contribuição deste estudo, tendo em vista que a maioria dos estudos anteriores analisou unicamente a dinâmica de curto prazo.

Por fim, uma simples simulação de três situações em cada um dos três regimes demonstrou que o retorno do fundo não pode ser explicado por $\beta_{1}$ e $\beta_{2}$ separadamente, mas sim a partir de sua conjugação. No Regime 0 (mais volátil) há a possibilidade de obter maior retorno do que no Regime 2 (menos volátil) em situações em que o retorno do mercado é positivo. Em compensação, quando o retorno do mercado é negativo, o retorno obtido no Regime 0 é inferior ao obtido no Regime 2, o que é esperado, tendo em vista que nos momentos de alta volatilidade acontecem as possibilidades de maiores e menores retornos. 
Pode-se elencar como limitações deste estudo o uso de dados consolidados e a restrição aos fundos de investimentos tradicionais. Para estudos futuros, sugere-se: realizar estimações separadas para cada categoria de fundos de investimento; incluir fundos com outros benchmarks, como Índice de Sustentabilidade Empresarial (ISE) e índices setoriais; incluir outros tipos de fundos, como Exchange Traded Funds (ETFs) e Fundos de Investimento Imobiliários (FIIs); utilizar outras proxies de mercado, como os índices IBrX, ISE e índices setoriais. Outra possibilidade para estudos futuros é utilizar como proxy índices internacionais.

\section{REFERÊNCIAS}

ALEXANDER, C.; Optimal Hedging Using Cointegration. Philosophical Transactions of the Royal Society, v. 357, n. 1358, p. 2039-2058, 1999.

ALEXANDER, C.; DIMITRIU, A. A Comparison of Cointegration and Tracking Error Models for Mutual Funds and Hedge Funds. ISMA Centre Discussion Papers in Finance, v. 4, p. 1-26, 2004.

ALEXANDER, C.; GIBLIN, I.; WEDDINGTON, W. Cointegration and asset allocation: a new active hedge fund strategy. Financial Risk and Financial Risk Management, v. 16, p. 65-89, 2002.

BALKE, N. S.; FOMBY, T. B. Threshold Cointegration. International Economic Review, p. 627-645, 1997.

CHU, P. K. K. The price linkages between the equity fund price levels and the stock markets: Evidences from cointegration approcach and causality analysis of Hong Kong Mandatory Provident Fund (MPF). International Review of Financial analysis, v. 19, n. 4, p. 281-288, 2010.

ENGLE, R. F.; GRANGER, C. W. J. Co-Integration and Error Eorrection: Representation, Estimation and Testing. Econometrica: Journal of the Econometric Society, v. 55, n. 2, p. 251-276, 1987.

GRANGER, C. W. J.; The Typical Spectral Shape of an Economic Variable. Econometrica: Journal of the Econometric Society, v. 34, n.1, p. 150-161, 1966.

GRANGER, C. W. J.; TERASVIRTA, T. Modelling Nonlinear Economic Relationships. Oxford University Press, capítulo 5, 1993.

GUJARATI, D. N. Econometria Básica. $4^{\mathrm{a}}$ ed. Tradução de Maria José Cylar Monteiro. Rio de Janeiro: Elsevier, 2006.

HAMILTON, J. A New Approach to the Economic Analysis of Nonstationary Time Series and the Business Cycle. Econometrica: Journal of the Econometric Society, v. 57, n. 2, p. 357-84, 1989.

JENSEN, M. C. The performance of mutual funds in the period of 1945-1964. Journal of Finance, v. 23, n. 2, p. 389-416, 1968. 
KIM, B. S. Linkages between the U.S. and the Asia-Pacific Exchange Traded Funds (ETF) Markets: Evidence from the 2007-2008 global financial crisis. Asian Academy of Management Journal of Accounting and Finance, v. 7, n. 1, p. 53-72, 2011.

LINTNER, J. Security prices, risk and maximal gains from diversification. The Journal of Finance, v. 20, n. 4, p. 587-615, 1965.

MARKOWITZ, H. Portfolio Selection. The Journal of Finance, v. 7, n.1, p. 77-91, 1952.

MAURER, T. A. Cointegration in Finance: An application to Index Tracking. SSRN, 2008.

SHARPE, W. F. Mutual Fund Performance. The Journal of business, v. 39, n. 1, p.119-138, 1966.

STOCK, J. H.; WATSON, M. W. Testing for Common Trends. Journal of American Statistical Association v. 83, n. 404, p. 1097-1107, 1988.

TONG, H. Threshold Models in Non-linear Time Series Analysis. Heidelberg: Springer Verlag, 1983.

TREYNOR, J. How to Rate Management of Investment Funds. Harvard Business Review, v. 43, n. 1, p. 63-75, 1965.

TSAY, R. S. Analysis of Financial Time Series. Third Edition. New Jersey: Wiley, 2010. 